\title{
DIVISORS ON SYMMETRIC PRODUCTS OF CURVES
}

\author{
ALEXIS KOUVIDAKIS
}

ABSTRACT. For a curve with general moduli, the Neron-Severi group of its symmetric products is generated by the classes of two divisors $x$ and $\theta$. In this paper we give bounds for the cones of effective and ample divisors in the $x \theta$ plane.

\section{INTRODUCTION}

Let $C$ be a smooth irreducible curve of genus $g, J(C)$ its Jacobian variety and $C_{d}$ its $d$-fold symmetric product. Fixing a point $P_{0}$ on $C$, we define the maps

$$
\begin{gathered}
u_{d}: C_{d} \rightarrow J(C) \text { by } u_{d}(D)=\mathscr{O}\left(D-d P_{0}\right), \\
i_{d-1}: C_{d-1} \rightarrow C_{d} \text { by } i_{d-1}(D)=D+P_{0} .
\end{gathered}
$$

On $J(C)$ we denote by $\boldsymbol{\theta}$, the theta divisor and by $\theta$, its class in the NeronSeveri group. On $C_{d}$ we denote by $\theta_{d}$, or simply (again) $\theta$, the class of $u_{d}^{*}(\theta)$ and by $x_{d}$, or simply $x$, the class of $i_{d-1}\left(C_{d-1}\right)$ in $C_{d}$. For curves $C$ with general moduli, it is known that the Neron-Severi group of the symmetric product $C_{d}$ is generated by $\theta$ and $x$, see [A-C-G-H]. In this paper we give estimates for the cones of the effective and ample divisors on $C_{d}$, in the $\theta, x$ plane.

By standard theory, we know the following things about the map $u_{d}$ :

1. Abel's Theorem. The fiber of the map $u_{d}$ containing the divisor $D$, is exactly the set of divisors belonging to the complete series of $D$.

2. JACOBI's InVERsion Theorem. The map $u_{g}: C_{g} \rightarrow J(C)$ is onto.

3. PoINCARE's Formula. We denote by $W_{d}$ the image of $C_{d}$ in the Jacobian by the map $u_{d}$ and by $w_{d}$ its class. For $0 \leq d \leq g$ we have

$$
w_{d}=\frac{\theta^{g-d}}{(g-d) !} .
$$

In particular, for $d=g-1$ we have $w_{g-1}=\theta$.

Received by the editors January 3, 1991.

1991 Mathematics Subject Classification. Primary 14H10, 14C20, 14J40; Secondary 14H35, $11 \mathrm{G} 30$.

Key words and phrases. Ample divisor, curve with general moduli, effective divisor, symmetric product of curve. 
4. If $d \leq g$, the map $u_{d}$ is birational to its image, reflecting the fact that $\mathbf{h}^{\mathbf{0}}(C, D)=1$, for $D$ general point in $C_{d}$.

If $g+1 \leq d \leq 2 g-2$, the generic fiber of $u_{d}$ has dimension $d-g$.

If $2 g-1 \leq d$, the map is a $\mathbf{P}^{d-g}$-fibration with tautological class $x$.

Lemma 1 (Intersections on $C_{d}$ ). For $0 \leq r \leq d \leq g$ we have on $C_{d}$

$$
\theta^{r} x^{d-r}=\frac{g !}{(g-r) !} \text {. }
$$

Proof. Indeed, $x=\operatorname{class}\left(i_{d-1}\left(C_{d-1}\right)\right)=\operatorname{class}\left\{D+P_{1}, D \in C_{d-1}, P_{1}\right.$ a fixed point in $C$ \} and so,

$$
x^{d-r}=\operatorname{class}\left\{D+P_{1}+\cdots+P_{d-r}, D \in C_{r}, P_{1}, \ldots, P_{d-r} \text { fixed in } C\right\} .
$$

Therefore $u_{d *} x^{d-r}=w_{r}$. Since the map $u_{d}$ is generically 1-1, projection formula implies that $\theta^{r} x^{d-r}$ on $C_{d}$, is equal to $\theta^{r} w_{r}$ on $J(C)$. Poincaré's Formula and the fact that $\theta^{g}=g$ ! complete the proof. Q.E.D.

We denote by $H_{a n}^{2 n}\left(C_{d}, \mathbf{Q}\right)$ the algebraic part of $H^{2 n}\left(C_{d}, \mathbf{Q}\right)$. Given an algebraic cycle $Z$ in $C_{d}$, we define the maps

$$
A_{k}: H_{a n}^{2 m}\left(C_{d}, \mathbf{Q}\right) \rightarrow H_{a n}^{2 m}\left(C_{d+k}, \mathbf{Q}\right)
$$

by

$$
A_{k}(Z)=\left\{E \in C_{d+k}, E-D \geq 0 \text { for some } D \in Z\right\}
$$

and

$$
B_{k}: H_{a n}^{2 m}\left(C_{d}, \mathbf{Q}\right) \rightarrow H_{a n}^{2 m-2 k}\left(C_{d-k}, \mathbf{Q}\right)
$$

by

$$
B_{k}(Z)=\left\{E \in C_{d-k}, D-E \geq 0 \text { for some } D \in Z\right\} .
$$

We have the standard formulas, see [A-C-G-H, p. 367].

Lemma 2 (Push-pull formulas for symmetric products).

$$
A_{k}\left(x^{\alpha} \theta^{\beta}\right)=\sum_{i=0}^{k}\left(\begin{array}{c}
\beta \\
i
\end{array}\right)\left(\begin{array}{c}
g-\beta+i \\
i
\end{array}\right)\left(\begin{array}{c}
d+k-\alpha-2 \beta \\
k-i
\end{array}\right) i ! x^{\alpha+i} \theta^{\beta-i}
$$

and

$$
B_{k}\left(x^{\alpha} \theta^{\beta}\right)=\sum_{j=0}^{k}\left(\begin{array}{c}
\alpha \\
k-j
\end{array}\right)\left(\begin{array}{c}
\beta \\
j
\end{array}\right)\left(\begin{array}{c}
g-\beta+j \\
j
\end{array}\right) j ! x^{\alpha-k+j} \theta^{\beta-j}
$$

\section{AMPLE AND EFFECTIVE DIVISORS}

We will use often the following criterion for ampleness, see [Ha]:

Lemma 3 (Nakai-Moishezon). Let $D$ be a Cartier divisor on a variety $X$. Then $D$ is ample on $X$, if and only if, for every subvariety $Y$ in $X$ of dimension $r$, we have that $D^{r} Y>0$.

In particular, when $X$ is a smooth surface this says that the cone of effective and the cone of ample divisors are dual under the intersection pairing.

If $X$ is a smooth surface, we have the following numerical criterion, for checking effectivity of a divisor $D$ on $X$, see [Ha]: 
Lemma 4. Let $X$ be a smooth surface, $H$ an ample divisor on $X$ and $D a$ divisor with $D^{2}>0$ and $D H>0$.

Then, for $n$ sufficiently large, $n D$ is an effective divisor.

Proof. We prove first that for $n$ large enough we have $\mathbf{h}^{2}(X, n D)=0$ : Indeed, $\mathbf{h}^{2}(X, n D)=\mathbf{h}^{\mathbf{0}}\left(X, K_{X}-n D\right)$, and if we assume that $\mathbf{h}^{2}(X, n D)>0$ for all $n$, then $K_{X}-n D$ is effective and so, $\left(K_{X}-n D\right) H>0$ (Lemma 3) i.e. $K_{X} H>$ $n D H$. Since $D H>0$, taking $n$ big enough-namely $n>K_{X} H$-we get $K_{X} H>n$. A contradiction. Therefore there exists an $n$ with $\mathbf{h}^{2}(X, n D)=0$ and the Riemann-Roch theorem completes the proof. Q.E.D.

In the higher dimensional case i.e. if $X$ is a smooth variety of dimension $d$, the Riemann-Roch theorem gives that

$$
\begin{aligned}
& \mathbf{h}^{\mathbf{0}}(X, D)-\mathbf{h}^{\mathbf{1}}(X, D)+\mathbf{h}^{2}(X, D)-\cdots+(-1)^{d} \mathbf{h}^{\mathbf{d}}(X, D) \\
& \quad=\frac{\mathbf{c}_{\mathbf{1}}^{d}(D)}{d !}+\left(\text { terms containing strictly lower powers of } \mathbf{c}_{\mathbf{1}}\right) .
\end{aligned}
$$

If $H$ is again an ample divisor on $X$ and $D$ a divisor satisfying $D^{r} H^{d-r}>0$ for all $0 \leq r \leq d$, then it is not known if

$$
\mathbf{h}^{\mathbf{0}}(X, n D)>0 \text { for } n \text { big enough }
$$

In order to have such a conclusion, we have to impose extra condition, for example, the restriction of $\mathscr{O}(D)$ on $H$ to be an ample divisor. We have

Theorem 1. Let $X$ be a d-dimensional variety, $H$ an ample effective divisor on $X$ and $D$ a divisor with $D^{d}>0$ and $\left.\mathscr{O}(D)\right|_{H}$ is an ample line bundle on $H$.

Then,

$$
\mathbf{h}^{\mathbf{0}}(n D)>0 \text { for } n \text { big enough. }
$$

Proof. We use the following lemmas:

Lemma 5 (Serre). Let $X$ be a proper scheme over a Noetherian ring. If $\mathcal{N}$ is an invertible sheaf on $X$, then the following conditions are equivalent:

(i) $\mathcal{N}$ is ample.

(ii) For each coherent sheaf $\mathscr{C}$ on $X$, there exists an integer $n_{0}$ depending on $C$ s.t. for each $i \geq 1$ and each $n \geq n_{0}$

$$
\mathbf{H}^{\mathrm{i}}\left(X, \mathscr{C} \otimes \mathscr{N}^{n}\right)=0 .
$$

Lemma 6. Let $S$ be a proper scheme over a Noetherian ring, $\mathscr{L}$ an ample line bundle on $S$ and $\mathscr{F}$ a line bundle generated by global sections. Then there exists an $n_{0}$ s.t.

$$
\forall n \geq n_{0} \quad \mathbf{H}^{\mathrm{i}}\left(S, \mathscr{L}^{n} \otimes \mathscr{F}^{k}\right)=0 \quad \forall k \geq 0, \quad \forall i \geq 1 .
$$

Acknowledgment. I would like to thank J.-F. Burnol, for showing me Lemma 6 above and the following proof.

Proof. We have an exact sequence $\bigoplus_{i=1}^{r} \mathscr{O}_{s} \rightarrow \mathscr{F} \rightarrow 0$. Tensoring by $\check{\mathscr{F}}$ we get $\bigoplus_{i=1}^{r} \check{\mathscr{F}} \rightarrow \mathscr{O}_{S} \rightarrow 0$. Hence (Koszul):

$$
0 \rightarrow(\check{\mathscr{F}})^{r} \rightarrow \bigoplus_{r}(\check{\mathscr{F}})^{r-1} \rightarrow \bigoplus_{\left(\begin{array}{l}
r \\
2
\end{array}\right)}(\check{\mathscr{F}})^{r-2} \rightarrow \cdots \rightarrow \bigoplus_{r} \check{\mathscr{F}} \rightarrow \mathscr{O}_{S} \rightarrow 0 .
$$


And so, tensoring by $\mathscr{F}^{r}$ we get

$$
0 \rightarrow \mathscr{O}_{S} \rightarrow \bigoplus_{r} \mathscr{F} \rightarrow \bigoplus_{\left(\begin{array}{l}
r \\
2
\end{array}\right)}(\mathscr{F})^{2} \rightarrow \cdots \rightarrow \bigoplus_{r}(\mathscr{F})^{r-1} \rightarrow(\mathscr{F})^{r} \rightarrow 0
$$

By Lemma 5 we can choose $n_{0}$ so that $\mathbf{H}^{\mathrm{i}}\left(S, \mathscr{L}^{n} \otimes \mathscr{F}^{k}\right)=0$ for all $i \geq 1$, $n \geq n_{0}, k=0,1, \ldots, r$. Then this $n_{0}$ works: suppose the claim is true for $k \leq l$. Tensoring the above exact sequence by $\mathscr{L}^{n} \otimes \mathscr{F}^{l+1-r}$, all sheaves have zero $\mathbf{H}^{\mathrm{i}}$ 's, $i \geq 1, n \geq n_{0}$, except maybe the last one which is $\mathscr{L}^{n} \otimes \mathscr{F}^{l+1}$. Therefore the last one has also zero $\mathbf{H}^{\mathrm{i}}$ 's, $i \geq 1, n \geq n_{0}$. Q.E.D.

Going back to the proof of Theorem 1, we use first the following fact: "A line bundle $L$ is ample on $H$ iff $L_{\text {red }}$ is ample on $H_{\text {red }}$ ". By this fact, we can replace $H$ by $m H$ without changing hypothesis on $D$, and so, we can assume that $H$ is in fact very ample on $X$. We denote by $L$ the line bundle $\mathscr{O}(D)$. We have that $L_{1}=L \otimes \mathscr{O}_{H}$ is ample on $H$. Let $H_{1}$ be the restriction of $\mathscr{O}(H)$ to $H$; then, $H_{1}$ is generated by global sections. Also $L_{1}$ is ample on $H$ and so, by the above Lemma 6 , there exists an integer $n_{0}$ s.t.

$$
\mathbf{h}^{\mathbf{i}}\left(H, L_{1}^{n} \otimes H_{1}^{k}\right)=0 \text { for all } i \geq 1, n \geq n_{0}, k \geq 0 .
$$

On the other hand $H$ is ample on $X$ and so, given the coherent sheaf $L^{n}$ there exists by Lemma 5 an integer $m_{n}$ s.t. $\mathbf{H}^{\mathrm{i}}\left(X, L^{n} \otimes H^{m_{n}}\right)=0$. Consider now the exact sequence:

$$
0 \rightarrow \mathscr{O}_{X}\left(L^{n} \otimes H^{(l-1)}\right) \rightarrow \mathscr{O}_{X}\left(L^{n} \otimes H^{l}\right) \rightarrow \mathscr{O}_{H}\left(L_{1}^{n} \otimes H_{1}^{l}\right) \rightarrow 0 .
$$

The corresponding long exact sequence gives

$$
\begin{aligned}
0 & \rightarrow \mathbf{H}^{\mathbf{0}}\left(X, L^{n} \otimes H^{(l-1)}\right) \rightarrow \mathbf{H}^{0}\left(X, L^{n} \otimes H^{l}\right) \rightarrow \mathbf{H}^{\mathbf{0}}\left(H, L^{n} \otimes H_{1}^{l}\right) \\
\rightarrow & \mathbf{H}^{\mathbf{1}}\left(X, L^{n} \otimes H^{(l-1)}\right) \rightarrow \mathbf{H}^{\mathbf{1}}\left(X, L^{n} \otimes H^{l}\right) \rightarrow \mathbf{H}^{\mathbf{1}}\left(H, L^{n} \otimes H_{1}^{l}\right) \\
\rightarrow & \mathbf{H}^{\mathbf{2}}\left(X, L^{n} \otimes H^{(l-1)}\right) \rightarrow \mathbf{H}^{2}\left(X, L^{n} \otimes H^{l}\right) \rightarrow \mathbf{H}^{2}\left(H, L^{n} \otimes H_{1}^{l}\right) \\
& \ldots \\
\rightarrow & \mathbf{H}^{\mathbf{k}}\left(X, L^{n} \otimes H^{(l-1)}\right) \rightarrow \mathbf{H}^{\mathbf{k}}\left(X, L^{n} \otimes H^{l}\right) \rightarrow \mathbf{H}^{\mathbf{k}}\left(H, L^{n} \otimes H_{1}^{l}\right)
\end{aligned}
$$

and so, we get for each $n \geq n_{0}$ that

$$
\mathbf{h}^{\mathbf{i}}\left(X, L^{n} \otimes H^{(l-1)}\right)=\mathbf{h}^{\mathbf{i}}\left(X, L^{n} \otimes H^{l}\right) \quad \text { for all } i \geq 2, l \geq 1 .
$$

Therefore, for each $n \geq n_{0}$ we have that

$$
\mathbf{h}^{\mathbf{i}}\left(X, L^{n}\right)=\mathbf{h}^{\mathbf{i}}\left(X, L^{n} \otimes H\right)=\cdots=\mathbf{h}^{\mathbf{i}}\left(X, L^{n} \otimes H^{m_{n}}\right)=0 \quad \text { for all } i \geq 2 .
$$

For each $n \geq n_{0}$ the Riemann-Roch theorem gives

$$
\begin{aligned}
\mathbf{h}^{0}\left(X, L^{n}\right)= & \mathbf{h}^{1}\left(X, L^{n}\right)+\frac{\mathbf{c}_{1}^{d}\left(L^{n}\right)}{d !} \\
& +\left(\text { terms containing strictly lower powers of } \mathbf{c}_{1}\right)
\end{aligned}
$$

and so, since $\mathrm{c}_{1}^{d}(L)>0$, we get that there exists an $n$ big enough s.t. $\mathbf{h}^{0}\left(X, L^{n}\right)$ $>0$. Q.E.D. 
3. The Class of The Diagonal AND of $\Gamma_{n}\left(g_{d}^{r}\right)$ 's

We recall some theory from [A-C-G-H]. Consider the diagonal map

$$
\phi_{a}=\phi: C_{d-2} \times C \rightarrow C_{d}
$$

defined by

$$
\phi(D, p)=D+2 p .
$$

The image of this map is the diagonal $\Delta_{d}$ in $C_{d}$. A special case of Proposition 5.1 on p. 358 in [A-C-G-H] gives that

Lemma 7 (MacDonald). The class $\delta_{d}$ of the diagonal $\Delta_{d}$ in $C_{d}$ is given by

$$
\delta_{d}=2((d+g-1) x-\theta) .
$$

We denote now by $g_{d}^{r}$ a base point free linear system of degree $d$ and dimension $r$ on $C$. Given such a $g_{d}^{r}$, then for each $n$ with $r<n \leq d$, we can construct in $C_{n}$ the following cycle

$$
\Gamma_{n}\left(g_{d}^{r}\right)=\left\{D \in C_{n} \text { s.t. } D \leq E \text { for some } E \in g_{d}^{r}\right\} .
$$

The standard way to calculate the class $\gamma_{n}\left(g_{d}^{r}\right)$ of the above cycle is given by the following lemma, see [A-C-G-H, Lemma 3.2, p. 342].

Lemma 8. For integers $d \geq n>r$ the class $\gamma_{n}\left(g_{d}^{r}\right)$ in $C_{n}$ is given by

$$
\gamma_{n}\left(g_{d}^{r}\right)=\sum_{k=0}^{n-r}\left(\begin{array}{c}
d-g-r \\
k
\end{array}\right) \frac{x^{k} \theta^{n-r-k}}{(n-r-k) !} .
$$

In the particular case where $n=r+1$ and so, $\Gamma_{r+1}\left(g_{d}^{r}\right)$ is a divisor in $C_{r+1}$, we can find the class as following:

We denote by $C^{\times(r+1)}$ the $(r+1)$ th Cartesian product of $C$, by $f_{1}, \ldots, f_{r+1}$ the class of the coordinate planes and by $\delta_{C}$ the class of the sum of the diagonals in the product. Also we define $\gamma_{C}=\pi^{*}\left(\gamma_{r+1}\left(g_{d}^{r}\right)\right)$, where $\pi: C^{\times(r+1)} \rightarrow C_{r+1}$ the canonical map. We have the following relations:

$$
\pi^{*} x=f \stackrel{\text { def }}{=} f_{1}+\cdots+f_{r+1}, \quad \pi^{*} \delta_{r+1}=2 \delta_{C}, \quad \delta_{r+1}=2((g+r) x-\theta) .
$$

Given a $g_{d}^{r}$ on $C$ we have a canonical map $\phi: C \rightarrow \mathbf{P}^{r}=\mathbf{P}$ and an induced (product) map $\Phi: C^{\times(r+1)} \rightarrow \mathbf{P}^{\times(r+1)}$. We denote by $\delta_{\mathbf{P}}$ the class of the sum of the diagonals in $\mathbf{P}^{\times(r+1)}$. Observe that

$$
\Phi^{*}\left(\delta_{\mathbf{P}}\right)=\delta_{C}+\gamma_{C} .
$$

We have $\delta_{\mathbf{P}}=f_{1}^{\mathrm{P}}+\cdots+f_{r+1}^{\mathbf{P}}$, where $f_{i}^{\mathrm{P}}$ 's are the classes of the coordinate planes in $\mathbf{P}^{\times(r+1)}$. Therefore, $\boldsymbol{\Phi}^{*}\left(\delta_{\mathbf{P}}\right)=\boldsymbol{\Phi}^{*}\left(f_{1}^{\mathbf{P}}+\cdots+f_{r+1}^{\mathbf{P}}\right)=d\left(f_{1}+\cdots+f_{r+1}\right)=d f$ and so, $\gamma_{C}=d f-\delta_{C}$.

Now, $\pi^{*}\left(\gamma_{r+1}\left(g_{d}^{r}\right)\right)=\gamma_{C}=d f-\delta_{C}=d \pi^{*} x-\frac{1}{2} \pi^{*}\left(\delta_{r+1}\right)$ and so, $\gamma_{r+1}\left(g_{d}^{r}\right)=$ $d x-\delta_{r+1} / 2$. Using relation (5) we conclude that

$$
\gamma_{r+1}\left(g_{d}^{r}\right)=\theta-(g-d+r) x .
$$

\section{FIRST BOUNDS FOR THE CONES}

We examine the case $d \leq g$. If $D$ is an effective divisor on $C_{d}$, then $u_{d}^{*} \theta^{d-1} \cdot D=\theta^{d-1} \cdot u_{d_{*}} D$, where $u_{d}$ the Abel-Jacobi map. Since $\theta$ is an ample 
divisor on $J(C)$, we get by Lemma 3 , that $\theta^{d-1} \cdot u_{d_{*}} D \geq 0$, where equality holds iff $u_{d_{*}} D=0$. This gives the first naive bound for the effective cone in $C_{d}$ :

Suppose that $D$ is divisor with class $a \theta-b x, a, b>0$ i.e. it "lies" in the fourth quarter of the $\theta, x$-plane. We define slope $m$ of $D$ to be $m=\frac{b}{a}$.

If $D$ is effective, then by the above discussion we have $(\theta-m x) \theta^{d-1} \geq 0$ which implies

$$
\frac{g !}{(g-d) !}-m \frac{g !}{(g-d+1) !} \geq 0 \text { i.e. } m \leq g-d+1 .
$$

If $D$ is ample, Lemma 3 implies that $(\theta-m x)^{d}>0$. Equivalently

$$
\sum_{k=0}^{d}\left(\begin{array}{l}
d \\
k
\end{array}\right) \theta^{k} m^{d-k} x^{d-k}>0,
$$

i.e.

$$
\sum_{k=0}^{d}\left(\begin{array}{l}
d \\
k
\end{array}\right) m^{d-k} \frac{g !}{(g-k) !}>0 .
$$

Since for $m=0$ this is positive, we must have $m<$ (min. posit. root of (7)).

For a divisor $D$ with class $a x-b \theta, a, b>0$, i.e. it "lies" in the second quarter of the $\theta, x$-plane we define the slope $\bar{m}$ of $D$ to be $\bar{m}=\frac{a}{b}$. Similar argument gives that if $D$ is effective then $\bar{m} \geq g-d+1$, and if $D$ is ample then $\bar{m}$ satisfies a similar relation as in (7). For example if $D$ is ample then for

$$
d=2 \text { we have } m<g-\sqrt{g} \text { and } \bar{m}>g+\sqrt{g} .
$$

5. EFFECTIVE AND AMPLE CONES FOR $C_{2}$

By the previous discussion we have the first bounds for the effective and ample cones for $C_{2}$. On the other hand using the Lemma 4 we know that every

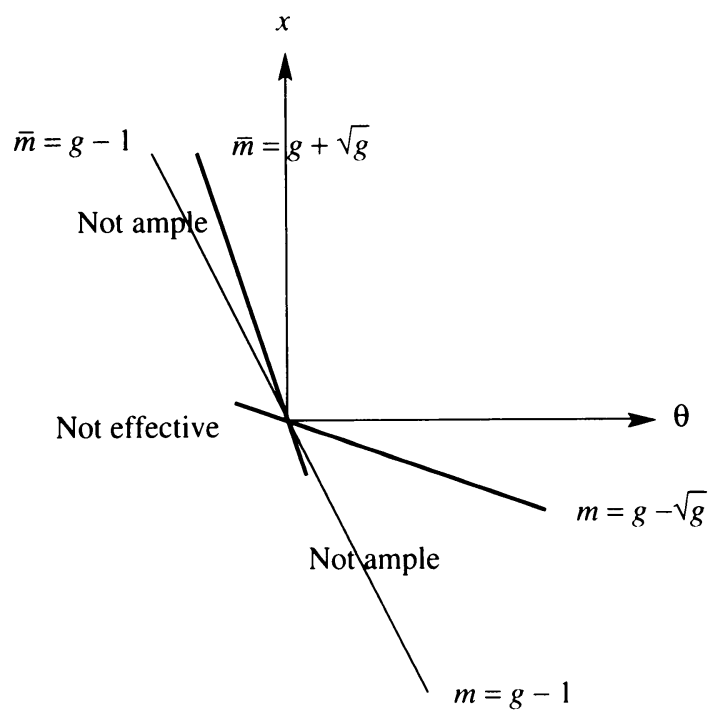

FIGURE 1. Bounds for the cones in $C_{2}$ 
class between the thick lines in Figure 1 is effective. Since the ample and effective cones are dual, it is enough to describe the effective cone. We make the observation:

Observation. If $D$ is an irreducible effective divisor with slope $m(\operatorname{resp} . \bar{m})$ between

$$
g-\sqrt{g}<m \leq g-1 \quad \text { (resp. } g+\sqrt{g}>\bar{m} \geq g-1),
$$

then it is unique with this property.

Indeed, if $D^{\prime}$ is another irreducible effective divisor with slope $m^{\prime}$ (resp. $\bar{m}^{\prime}$ ) in the above range then we get $D D^{\prime}<0$, a contradiction.

If we are able to find such a divisor, then this describes the cone. In the second quarter such a divisor exists, namely the diagonal: Recall that $\delta_{2}=$ $2((g+1) x-\theta)$ and so $\bar{m}=g+1$ i.e. $g+\sqrt{g}>\bar{m}>g-1$. Therefore the slope of the effective cone in the second quarter is given by

$$
\bar{m}_{e f}=g+1
$$

and (by duality) of the ample cone by

$$
\bar{m}_{a}=2 g \text {. }
$$

(Note that a divisor with slope $\bar{m}_{a}$, has positive intersection with all the effective divisors in the first or the fourth quarter.)

Also, if $C$ is of genus 2 , then it is hyperelliptic and the corresponding $g_{2}^{1}$ gives an effective class in $C_{2}$ belonging in the fourth quarter with slope $m=1$, see formula (6). By the above observation we get that $m_{e f}=1$ and by duality $m_{a}=0$. For general genus, we have the following:

Theorem 2. Let $C$ be a curve of genus $g \geq 3$ with general moduli. For the slopes of the cones in $C_{2}$, in the fourth quarter of the $\theta, x$-plane, we have

1. If $g$ is a square, then $m_{e f}=m_{a}=g-\sqrt{g}$. so

2. If $g$ is not a square and $g>3$, then $g-[\sqrt{g}]+1 \geq m_{e f} \geq g-\sqrt{g}$ and

$$
g-\sqrt{g} \geq m_{a} \geq g-\frac{g}{[\sqrt{g}]-1} .
$$

3. If $g=3$, then $m_{e f}=\frac{4}{3}$ and $m_{a}=\frac{6}{5}$.

Proof. We use degenerations to special curves: From the formula (6) we have that the class of $\gamma_{2}\left(g_{d}^{1}\right)$ in $C_{2}$ is given by

$$
\gamma_{2}\left(g_{d}^{1}\right)=\theta-(g-d+1) x .
$$

Therefore if $d<\sqrt{g}+1$, the slope of the divisor is

$$
m^{0}=g-d+1 \geq g-\sqrt{g} .
$$

Take a smooth curve $C^{0}$ with a $g_{d}^{1}, d<\sqrt{g}+1$. If in addition, we choose the curve to be "general" having such a $g_{d}^{1}$, then the corresponding divisor $\Gamma_{2}\left(g_{d}^{1}\right)$ is irreducible in $C_{2}$. Since $C^{0}$ is special, the $H_{a n}^{*}\left(C^{0}, \mathbf{Q}\right)$ may not be generated by $x, \theta$. Consider in $H_{a n}^{*}\left(C^{0}, \mathbf{Q}\right)$ the plane $\Pi$ spanned by $x, \theta$. By the previous analysis, since $m^{0}>g-\sqrt{g}$ the intersection of the effective cone with the plane $\Pi$ is given by the slopes $\bar{m}_{e f}^{0}=g+1$ and $m_{e f}^{0}=g-d+1$.

Since $\mathscr{M}_{g}$ is connected, we can find a flat family of smooth curves $\mathscr{C} \rightarrow \Delta$, $\Delta$ a disk, with central fiber $C^{0}$ and the other fiber curves with general moduli. 


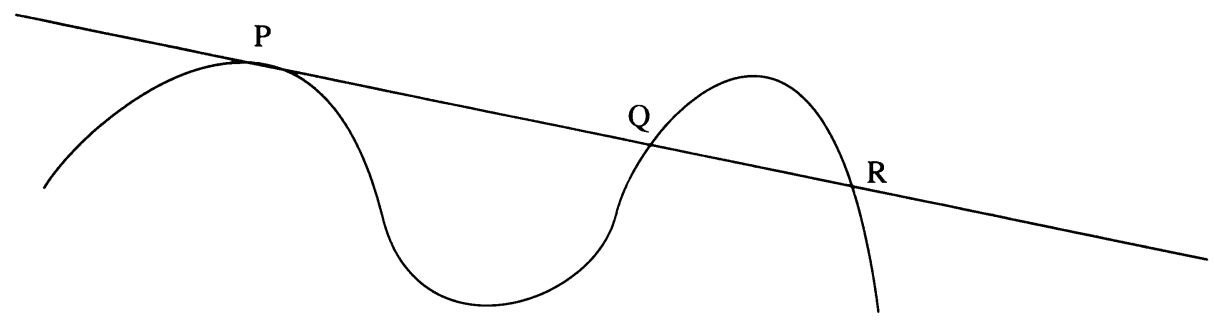

Figure 2. The genus 3 case

Let $\Phi: \mathscr{C}_{2} \rightarrow \Delta$ be the flat family with fibers the 2-symmetric products of the fibers of $\mathscr{C} \rightarrow \Delta$.

Suppose that the general curve has an irreducible divisor with slope $m, g-$ $1 \geq m>g-\sqrt{g}$. By the above observation, this is unique and so, it gives rise to an effective divisor in $\mathscr{C}_{2} \backslash \Phi^{-1}(0)$. Degenerating to the central fiber $C_{2}^{0}$, we get an effective divisor $D^{0}$. Since the degeneration "preserves" the algebraic equivalence we have that $D^{0}$ belongs to $\Pi$ and so, since it is effective, the slope $m$ satisfies $g-d+1 \geq m \geq g-\sqrt{g}$.

Therefore if $g$ is a square i.e. $g=k^{2}$, then choosing $d=k-1=\sqrt{g}-1$ we get that $m$ has to satisfy $m \leq g-\sqrt{g}$.

Since for slopes smaller than $g-\sqrt{g}$ we are in the effective cone by Lemma 4 , we conclude that

$$
m_{e f}=m_{a}=g-\sqrt{g} .
$$

If $g$ is not a square, choosing $d=[\sqrt{g}]$ we have

$$
g-\sqrt{g} \leq m_{e f} \leq g-[\sqrt{g}]+1 .
$$

The estimation of $m_{a}$ comes from the duality of the ample and effective cone. Of course as $g \rightarrow \infty$ we have $m_{e f} \sim m_{a} \sim g-\sqrt{g}$. Q.E.D.

The case $g=3$. In this case we can obtain for the general curve an irreducible divisor with slope $\frac{4}{3}$. (See Figure 2.) It is known that a general smooth curve $C$ of genus 3 , can be represented as a smooth plane quartic. We construct the following divisor in $C_{2}$ : For each point $\mathrm{P}$ in $C$ consider the tangent at that point. This intersects the curve at two additional points $Q, R$. Take now in $C_{2}$ the divisor $D$ consisting of all the sums $\mathrm{Q}+\mathrm{R}$ (with $\mathrm{P}$ moving on $C$ ). In order to calculate the class of $D$, we find its intersections with $x$ and $\delta_{2}$.

Intersection with $x$. The degree of the dual curve is 12 . Fixing a point $\mathrm{Q}$ on $C$, we ask for the number of tangents to the curve passing through $\mathrm{Q}$ (excluding the tangent to the curve at $\mathrm{Q}$ ). These are 10 . So $D x=10$.

Intersection with $\delta_{2}$. This is twice the number of bitangents to $C$, so $D \delta_{2}=$ $28 \times 2=56$.

Therefore if $D \sim a \theta-b x$ then, $D x=10$ i.e. $3 a-b=10$ and $D \theta=56$ i.e. $2(a \theta-b x)(4 x-\theta)=56$ so $6 a-b=28$. This gives $a=6, b=8$, i.e. $m_{e f}=\frac{4}{3}, m_{a}=\frac{6}{5}$.

\section{ONE SIDE SLOPE OF EFFECTIVE CONE IN $C_{d}$}

Theorem 3. The boundary of the effective cone in $C_{d}$, in the second quarter of the $\theta, x$-plane, is given by the class $\delta_{d}$ of the diagonal $\Delta_{d}$

$$
\delta_{d}=2((d+g-1) x-\theta) \text { i.e. } \bar{m}_{e f}=d+g-1 .
$$


Proof (Induction on $d$ ). For $d=2$ it has been proved. Assuming it is true for $d$, we prove it for $d+1$, i.e. we prove that in $C_{d+1}, \bar{m}_{e f}=d+g$.

Suppose that there exists an irreducible divisor $D$ on $C_{d+1}$ with class $m x_{d+1}$ $-\theta_{d+1}$ and $m<d+g$ (we add subindices for avoiding confusion). Fixing a point in $C$, we define the canonical embedding $i_{k}: C_{k} \rightarrow C_{d+1}$. Note that $i_{k}^{*}\left(x_{d+1}\right)=x_{k}$ and $i_{k}^{*}\left(\theta_{d+1}\right)=\theta_{k}$. The image of $C_{d}$ is an ample divisor on $C_{d+1}$, see [A-C-G-H, p. 310] and so, $i_{d}^{*}(D)$ is effective nonzero on $C_{d}$. Therefore the slope must satisfy $m \geq d+g-1$.

Since $\Delta_{d+1}, D$ are irreducible the intersection $\Delta_{d+1} D$ is nonempty effective. Indeed, $D, \Delta$ are not disjoint. Otherwise,

$$
m(d+g) x_{d+1}^{2}-(m+d+g) \theta_{d+1} x_{d+1}+\theta_{d+1}^{2}=0 .
$$

Applying $i_{2}^{*}$ we get $m(d+g) x_{2}^{2}-(m+d+g) \theta_{2} x_{2}+\theta_{2}^{2}=0$. Then formula (1) implies $m d=(d+1) g$. Since $i_{2}^{*}\left(\Delta_{d+1}\right)$ contains $\Delta_{2}, \Delta_{2}$ must be disjoint from $i_{2}^{*}(D)=D_{2}$. Therefore, $\Delta_{2} D_{2}=0$ i.e. $\left(m x_{2}-\theta_{2}\right) \delta_{2}=0$ i.e. $m=2 g$, and the above relation becomes $2 g d=(d+1) g$ i.e. $d=1$ a contradiction.

Therefore $B_{1}\left(D \Delta_{d+1}\right)$, see $\S 1$ for definition of $B_{1}$, lies in the effective cone of $C_{d}$, i.e.

$$
\text { slope of } B_{1}\left(D \Delta_{d+1}\right) \geq d+g-1 \text {. }
$$

Now,

$$
\operatorname{class}\left(D \Delta_{d+1}\right)=m(d+g) x_{d+1}^{2}-(m+d+g) x_{d+1} \theta_{d+1}+\theta_{d+1}^{2} .
$$

By Lemma 2 we have

$$
B_{1}\left(x_{d+1}^{2}\right)=2 x_{d}, \quad B_{1}\left(x_{d+1} \theta_{d+1}\right)=\theta_{d}+g x_{d}, \quad B_{1}\left(\theta_{d+1}^{2}\right)=2(g-1) \theta_{d} .
$$

Therefore,

$$
B_{1}\left(D \Delta_{d+1}\right)=\left(2 m d+m g-d g-g^{2}\right) x_{d}-(m+d-g+2) \theta_{d} .
$$

Since $m \geq d+g-1$, both coefficients are positive and so,

$$
\text { slope of } B_{1}\left(D \delta_{d+1}\right)=\frac{2 m d+m g-d g-g^{2}}{m+d-g+2} \text {. }
$$

Relation (11) implies that

$$
\frac{2 m d+m g-d g-g^{2}}{m+d-g+2} \geq d+g-1
$$

i.e. $m(d+1) \geq d g+d^{2}+d+3 g-2$. Since $m<d+g$ we get $(d+g)(d+1)>$ $d g+d^{2}+d+3 g-2$ or $1>g$, a contradiction. Q.E.D.

\section{BOUNDS FOR THE EFFECTIVE CONE IN $C_{r}, r \geq 3$}

We start with $C_{3}$. Let $D$ be a divisor in $C_{3}$ with class $\theta-m x, m \geq 0$, i.e. it "lies" in the fourth quarter. Since $x$ is the class of $i_{2}\left(C_{2}\right)$ in $C_{3}$ and

we conclude that

$$
\frac{d}{d m}(\theta-m x)^{3}=-3(\theta-m x)^{2} x
$$

$$
\frac{d}{d m}(\theta-m x)^{3}=0 \text { in } C_{3} \quad \Leftrightarrow \quad(\theta-m x)^{2}=0 \text { in } C_{2},
$$

i.e. when $m=g+\sqrt{g}$ or $m=g-\sqrt{g}$. 




FIGURE 3. The graph of $(\theta-m x)^{3}$ 3.

The graph of the $(\theta-m x)^{3}$ considered as function of $m$ is given by Figure

Theorem 4. We denote by $r_{1}$ the root of $(\theta-m x)^{3}=0$ closest to 0 . Then for $m<r_{1}$, the class $\theta-m x$ is effective.

Proof. Note that this equation has three positive roots, see Figure 3 above. Approximately as $g \rightarrow \infty, r_{1}$ goes to $g-\sqrt{3 g}$. Say $D$ a divisor with class $\theta-m x$ where $m<r_{1}$. By Theorem 2 the restriction of $D$ to $C_{2}$ is ample. Since $D^{3}>0$, Theorem 1 , applied to $H=C_{2}$, gives the result. Q.E.D.

It is difficult to continue the above method for higher $r$ 's, since we do not have a good estimate for the ample cone in $C_{3}$. For these cases we have

Theorem 5. Let $C$ be a smooth curve of genus $g \geq 1$ with general moduli. For $r \geq 3$ we have the following estimates for the effective and ample cone of $C_{r}$ in the fourth quarter of the $\theta, x$-plane

1. If $r \geq g+1$ then the boundary of the effective and ample cone is given by the $\theta$ line.

2. If $3 \leq r \leq g$ then we have the following bounds for the effective cone.

Bound from inside: for each rational $m$ with

$$
0 \leq m \leq \max \left(\left[\frac{g}{r}\right], g-(r-1) \sqrt{g}\right)
$$

there is an effective divisor with slope $m$.

Bound from outside: for each $m$ with $m>g-[\sqrt{r-1} \sqrt{g}]$ there is no effective divisor with slope $m$.

Remarks. 1. For $r=g$ the slope of the boundary of the effective cone is equal to 1 . Indeed in this case $\left[\frac{g}{r}\right]=1=g-[\sqrt{r-1} \sqrt{g}]$.

2. For $r \leq \sqrt{g}$ we have that

$$
\max \left(\left[\frac{g}{r}\right], g-(r-1) \sqrt{g}\right)=g-(r-1) \sqrt{g} .
$$

For $r \geq \sqrt{g}$ we have that

$$
\max \left(\left[\frac{g}{r}\right], g-(r-1) \sqrt{g}\right)=\left[\frac{g}{r}\right] .
$$

Proof. The proof of the first part of the theorem is easy: For $r \geq g+1$ we have that $\theta^{r}=u_{r}^{*}(\theta)^{r}=0$ and so, the class $\theta$ is not ample. On the other hand, since $\theta$ is ample on the Jacobian and $x$ is ample on $C_{r}$, see [A-C-G-H, p. 310], projection formula and Lemma 3, imply that the class $\theta+\varepsilon x$ is ample, for each $\varepsilon>0$. Therefore the bound for the ample cone is given by the line $\theta$. Also any divisor with class $\theta-\sigma x$ cannot be effective since there is an $\varepsilon>0$ small enough with $(\theta+\varepsilon x)^{r-1}(\theta-\sigma x)<0$; a contradiction by Lemma 3 . Therefore the bound of the effective cone is given by the $\theta$ line too.

To prove the second part of the theorem we use again degenerations to special curves. We start with a lemma: 
Lemma 9. If a curve $C$ has a "general" $g_{d}^{r-1}, r \geq 3$ (i.e. without base points and not composed with an involution), then for any $d \geq r$ the divisor $\Gamma_{r}\left(g_{d}^{r-1}\right)$ in $C_{r}$ is irreducible.

Proof. This is an application of the fact that the monodromy acts as the full symmetric group on the generic divisor of $g_{d}^{r}$. Q.E.D.

Let us now do some calculations. Recall from relation (6), that the class of $\gamma_{r}\left(g_{d}^{r-1}\right)$ is given by $\theta-(g-d+r-1) x$. Formula (1) gives that

$$
x^{k} \theta^{r-k}=\frac{g !}{(g-r+k) !} \quad \text { and } \quad x^{k+1} \theta^{r-k-1}=\frac{g !}{(g-r+k+1) !} .
$$

Using Lemma 8 we have for $d+1 \geq 2 r$ that

$$
\gamma_{r}\left(g_{d-r+2}^{1}\right)=\sum_{k=0}^{r-1}\left(\begin{array}{c}
d-r-g+1 \\
k
\end{array}\right) \frac{x^{k} \theta^{r-k-1}}{(r-k-1) !} .
$$

Therefore intersection number $I=\gamma_{r}\left(g_{d}^{r-1}\right) \cdot \gamma_{r}\left(g_{d-r+2}^{1}\right)$ is

$$
\begin{aligned}
I= & \sum_{k=0}^{r-1}\left(\begin{array}{c}
d-r-g+1 \\
k
\end{array}\right) \frac{g !}{(g-r+k) !(r-k-1) !} \\
& -(g-d+r-1) \sum_{k=0}^{r-1}\left(\begin{array}{c}
d-r-g+1 \\
k
\end{array}\right) \frac{g !}{(g-r+k+1) !(r-k-1) !} \\
= & g \sum_{k=0}^{r-1}\left(\begin{array}{c}
d-r-g+1 \\
k
\end{array}\right)\left(\begin{array}{c}
g-1 \\
r-k-1
\end{array}\right) \\
& -(g-d+r-1) \sum_{k=0}^{r-1}\left(\begin{array}{c}
d-r-g+1 \\
k
\end{array}\right)\left(\begin{array}{c}
g \\
r-k-1
\end{array}\right) \\
= & g\left(\begin{array}{c}
d-r \\
r-1
\end{array}\right)-(g-d+r-1)\left(\begin{array}{c}
d-r+1 \\
r-1
\end{array}\right) \\
= & A\left(d^{2}-2 d(r-1)-(r-1)(g-r-1)\right) \quad(A \text { a positive constant }) .
\end{aligned}
$$

This implies that

$$
I \leq 0 \Leftrightarrow r-1-\sqrt{r-1} \sqrt{g} \leq d \leq r-1+\sqrt{r-1} \sqrt{g} .
$$

Take now a smooth curve $C^{0}$ having a "general" $g_{d_{0}}^{r-1}$ with $d_{0}=r+1+$ $[\sqrt{r-1} \sqrt{g}]$ (note that $d_{0} \geq r$ ). We claim that the irreducible divisor $D=$ $\Gamma_{r}\left(g_{d_{0}}^{r-1}\right)$ with class $\gamma_{r}\left(g_{d_{0}}^{r-1}\right)=\theta-(g-[\sqrt{r-1} \sqrt{g}]) x$ gives the bound for the effective cone in the $\theta, x$-plane for $\left(C^{0}\right)_{r}$. Indeed, note first that the divisor $D$ is covered by a family of curves $\mathscr{C}_{d_{0}-r+2}$ with class $\gamma_{r}\left(g_{d_{0}-r+2}^{1}\right)$. These curves correspond to the various $g_{d-r+2}^{1}$ 's obtained by the one-parameter family of hyperplane sections of the image of the curve in $\mathbf{P}^{r-1}$, through collections of $r-2$ fixed points on this curve. Suppose that there exists another irreducible effective divisor $D^{\prime}$ with slope $m^{\prime}$ strictly greater than the slope of $D$. Then relation (13) implies that $D^{\prime} \cdot \gamma_{r}\left(g_{d_{0}-r+2}^{1}\right)<0$ and so, since $D^{\prime}$ is irreducible we get that all the members of $\mathscr{C}_{d_{0}-r+2}$ are contained in $D^{\prime}$. But since the divisor 
$D^{\prime}$ is "covered" by the family $\mathscr{C}_{d_{0}-r+2}$ this implies that $D$ is contained in $D^{\prime}$. A contradiction. The rest of the proof for the bound from outside goes, using degenerations after a possible base change, as the proof of Theorem 2 .

To prove the case for the bound from inside, we use the maps $A_{k}$ defined in the introduction of this paper. From formula (2) we have

$$
A_{r-2}\left(x_{r}\right)=(r-1) x_{2} \text { and } A_{r-2}\left(\theta_{r}\right)=\theta_{2}+g(r-2) x_{2} .
$$

Since in $C_{2}$ the slope $m$ with $m<g-\sqrt{g}$ is "effective", pulling back by the $A_{r-2}$ map we get in $C_{r}$ that

$$
A_{r-2}\left(\theta_{r}-(g-\sqrt{g}) x_{r}\right)=\theta_{2}-(g-(r-1) \sqrt{g}) x_{2} .
$$

On the other hand we have that for $d=g+r-1-\left[\frac{g}{r}\right]$, a general curve has a $g_{d}^{r-1}$ (this is the minimum $d$ for which the Brill-Noether number $\rho$ is nonnegative). Using formula (6) we obtain the following class of an effective divisor in $C_{r}$ :

$$
\gamma_{r}\left(g_{d}^{r-1}\right)=\theta-\left[\frac{g}{r}\right] x
$$

and this concludes the proof. Q.E.D.

\section{ACKNOWLEDGMENT}

This is part of the author's thesis at Harvard University. I would like to thank Joe Harris for proposing the problem and for his help during the preparation of this work.

\section{REFERENCES}

[A-C-G-H] E. Arbarello, M. Cornalba, P. Griffiths, and J. Harris, Geometry of algebraic curves. I, Springer, 1985.

[Ha] R. Hartshorne, Algebraic geometry, Springer, 1977.

Department of Mathematics, University of Pennsylvania, David Rittenhouse Laboratory, Philadelphia, Pennsylvania 19104-6395

E-mail address: alexk@pennsas.upenn.edu 